\title{
Type 1 Diabetes in Children: The Bahraini Dilemma
}

\author{
K. Hart ${ }^{1}$, Y. Nicolaidou ${ }^{1}$, A. O. Musaiger ${ }^{2}$, S. M. Alqallaf ${ }^{3}$, F. Al-Haddad ${ }^{4}$ \\ ${ }^{1}$ Department of Nutritional Science, University of Surrey, Guildford. GU2 $7 \mathrm{XH},{ }^{2}$ Nutrition and Health Studies Unit and \\ ${ }^{3}$ College of Health Sciences, University of Bahrain, Bahrain and ${ }^{4}$ Salmaniya Medical Complex Dietetic Department,
}

Manama, Bahrain

In the Middle East approximately one in four adult deaths is attributed to diabetes ${ }^{(1)}$. Bahrain has not escaped the many challenges and consequences of diabetes with rates of childhood Type 1 diabetes mellitus (T1DM) more than doubling in the past ten years ${ }^{(2)}$. Whilst a healthy diet and lifestyle is important in maintaining good blood glucose control and preventing long-term complications ${ }^{(3)}$ little or no data exists on the dietary intake or lifestyle of children living with T1DM in Bahrain. As part of an ongoing project characterising the Bahraini paediatric T1DM population, this paper presents data on the social and medical history of children with T1DM and healthy controls, in an attempt to elucidate the potential aetiology of the disease.

Ethical approval was received from the Health Ethics and Research Committee of the Salamaniya Medical Complex in Bahrain, to undertake a descriptive case-control study of all children aged 6-12 years old receiving a confirmed diagnosis of T1DM in the years 2009 and 2010, compared with a matched control group of children recruited from primary health care centres. Data for cases was extracted from the Diabetes Registry Forms (DRF) completed for all newly diagnosed patients. These record the family's socio-demographic status, mother's pre and postnatal history, infant feeding practices, and the child's past medical history and family history of diabetes. Comparative data for cases was collected using an adapted version of the DRF administered by the local researcher. Height and weight of all children were measured using standardized methods at diagnosis/ recruitment. PASW (v19) was used to compare the characteristics of the cases and controls using independent $t$ tests, Mann Whitney U tests and Chi Squared analysis as appropriate.

The data for 59 cases and 60 controls were available for analysis. The cases and controls were well matched for age, gender and body mas index (BMI) (Mean age 9.66[1.72] and 8.67[2.01] years; 49\% and 46\% male; mean BMI 16.9[5.1] and $17.3[4.7] \mathrm{kg} / \mathrm{m}^{2} \mathrm{respectively)}$. A significant association between economic situation and diabetes diagnosis was identified $(p=0.04)$ but this was due to more cases reporting their income as 'moderate' rather than a skew towards the 'high' or 'low' income bands. Children with diabetes were less likely to be Bahraini nationals $(p<0.001)$ but there was no significant difference in self-reported race with the majority of all children described as of Arab origin. A family history of T1DM was rarely reported, but cases were more likely to have a mother with T2DM $(p=0.03)$, gestational diabetes $(p=0.03)$ and gestational hypertension $(p=0.06)$ and, prior to diabetes diagnosis, to have suffered from other childhood illness $(p<0.001)$, most commonly tonsillitis, or to have undergone surgery $(p=0.004)$. No significant association between T1DM diagnosis and infant feeding was seen with over $90 \%$ of all children having received some breast milk and $59 \%$ and $65 \%$ of cases and controls respectively still receiving breast milk beyond 6 months.

The aetiology of T1DM likely involves an interaction between genetic and environmental factors. Although unable to fully interrogate potential genetic differences this analysis provides support for the role of infections as an environmental trigger for T1DM ${ }^{(4)}$. Whilst a role for infant feeding practices could not be confirmed, analyses of dietary intakes and Vitamin D status in this population group are ongoing and will further inform the development of effective prevention and management strategies to tackle paediatric T1DM in the Middle East.

1. WHO (2004) Diabetes Action Now. Switzerland: WHO

2. Pediatric Endocrine \& Diabetes Healthcare Team (2008) Statistic on Type 1 Diabetes in Bahrain. Juffair: Bahrain Ministry of Health.

3. Rovner AH, Nansel TR (2009) Diab Ed 35, 97-107.

4. Galleri L, Sebastiani G, Vendrame F et al. (2012) Adv Exp Med Biol 771, 252-71. 